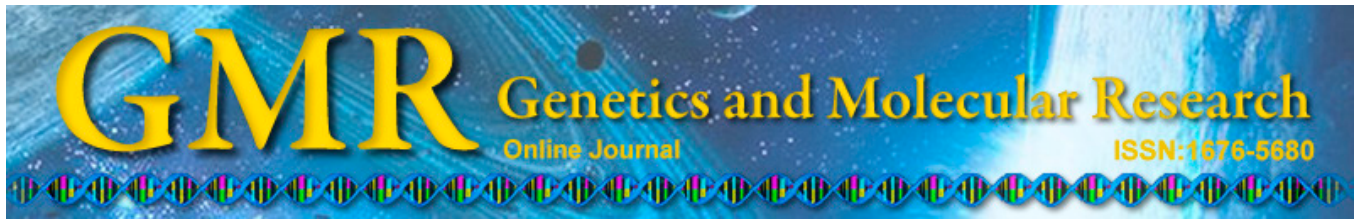

\title{
Cumulative methylation alternations of gene promoters and protein markers for diagnosis of epithelial ovarian cancer
}

\author{
B.L. Xing, T. Li, Z.H. Tang, L. Jiao, S.M. Ge, X. Qiang and J. OuYang \\ Department of Pathology, Changzhou Maternal and Child Health Care Hospital, \\ Changzhou, China \\ Corresponding author: B.L. Xing \\ E-mail: 793506861@qq.com
}

Genet. Mol. Res. 14 (2): 4532-4540 (2015)

Received July 3, 2014

Accepted December 11, 2014

Published May 4, 2015

DOI http://dx.doi.org/10.4238/2015.May.4.11

\begin{abstract}
DNA methylation plays an important role in carcinogenesis and cancer development. In this study, we examined gene methylation in epithelial ovarian cancer (EOC) using cationic conjugated polymer (CCP)-based fluorescence resonance energy transfer (FRET) to evaluate the application of cumulative methylation alternations of genes associated with cancer antigen 125 for early cancer diagnosis. The methylation status of 3 genes (Ras association domain family 1 isoform A, RASSF $1 A$; opioid-binding protein/cell adhesion molecule, OPCML; homeobox A9, HOXA9) were examined and compared in $35 \mathrm{EOC}$ samples and 11 normal ovarian tissue samples using CCP-based FRET. Gene methylation levels were clustered into 3 sections and assigned a value; values for the 3 genes were summed. Although methylation of the $O P C M L$ gene was significantly associated with stage, histological types, grade, and ascites and that of RASSF1A and $H O X A 9$ was not, the sum for the 3 genes was significantly associated with stage and ascites. The sum showed higher sensitivity (85.7\%) and specificity (100\%) for discriminating EOC from normal ovarian tissues than did the methylation status of RASSF1A, OPCML, and HOXA9
\end{abstract}


(48.6, 77.1, 77.1, and 100, 88.1, 100\%, respectively). Combining cancer antigen 125 levels with the sum increased the sensitivity to $94.3 \%$. The detection and analysis of a panel of genes' methylation status with the CCP-based FRET technique may be useful for diagnosis and screening of EOC; the associated cancer antigen 125 can be used to increase diagnostic sensitivity.

Key words: Cancer antigen 125; Methylation level detection; Cationic conjugated polymer-based fluorescence resonance energy transfer; Epithelial ovarian cancer; DNA methylation

\section{INTRODUCTION}

Epithelial ovarian cancer (EOC) is the most lethal gynecologic malignancy. Because of the lack of early diagnosis methods and no obvious symptoms, EOC, which accounts for $90 \%$ of ovarian cancer, is diagnosed at an advanced stage (FIGO III and IV) in $75 \%$ of all cases, when the disease has spread throughout the abdomen. Patients with advanced-stage disease have a 5 -year survival rate of only $30 \%$ in contrast to early-stage disease confined to the ovaries, for which 5-year survival rate exceeds $80 \%$ (Jemal et al., 2010). The overall survival of women with ovarian cancer has not changed in over 50 years (Kurman, 2013). Over the past 2 decades, numerous studies, including large clinical trials, have been conducted in an effort to reduce mortality by developing screening tests such as serum cancer antigen 125 (CA125) and transvaginal ultrasound to detect early-stage ovarian cancer that is confined to the ovaries. Unfortunately, these methods show high false-negative rates, as well as low sensitivity and specificity in the clinic, and have not demonstrated the capacity to reduce population morbidity and/or mortality (Menon and Jacobs, 2000; Jacobs and Menon, 2004; Munkarah et al., 2007).

DNA methylation is an important epigenetic modification that affects gene expression without changing the DNA sequence (Egger et al., 2004). During cancer development, cells undergo profound alterations in DNA methylation patterns, with functional consequences on the activity of key genes intimately involved in the carcinogenic process (Bast Jr. et al., 2005). Recent studies have shown that aberrant DNA methylation, which typically occurs before patients develop clinical manifestations and radiographic evidence, provides a new molecular approach for the early diagnosis of cancer (Issa, 2007; Zhu and Yao, 2007). The DNA methylation profile of a large number of genes have been associated with the molecular, clinical, and pathological features of ovarian cancer (Widschwendter et al., 2009; Cul'bová et al., 2011; Ho et al., 2012).

It is likely that determining the methylation status of a panel of genes, rather than an individual gene, will result in more sensitive and specific diagnosis (Montavon et al., 2012; Bhagat et al., 2012). We developed a cationic conjugated polymer (CCP)-based fluorescence resonance energy transfer (FRET) method for detecting the methylation levels of Ras association domain family 1 isoform A (RASSF 1A), opioid-binding protein/cell adhesion molecule $(O P C M L)$, and homeobox A9 (HOXA9) of EOCs and associated analysis of DNA methylation (Zhang et al., 2014). We examined the correlation between the data generated by our technique and the clinical and pathological features of EOC. The study provides a rational basis for the development of a protocol for the diagnosis and screening of EOC. 


\section{MATERIAL AND METHODS}

\section{Sample collection and gene selection}

Based on a retrospective study, 35 EOC samples and 11 normal ovarian samples were obtained from the Changzhou Maternal and Child Health Care Hospital from 2004-2011. All patients provided consent and the study was approved by the hospital Ethics Committee. Histologic classification of EOC was conducted according to the World Health Organization criteria, and tumor stages were established according to the International Federation of Gynecology and Obstetrics. Because high-grade serous ovarian cancer is the subtype with highest prevalence, estimated at approximately $70 \%$ of all cases (Landen Jr. et al., 2008), the grade of differentiation was defined as high-grade serous cancers and other grades. Eleven cases of normal ovarian tissues were collected from patients without cancer who underwent salpingo-oophorectomy at the time of surgery for benign gynecological diseases. Clinical characteristics, including age and preoperative CA125 levels, and pathological characteristics, including histopathologic diagnosis, grade, and stage, were determined for each patient. The promoter of the selected gene was expected to be unmethylated in normal cases and methylated in EOC cases.

\section{DNA preparation}

Genomic DNA samples of formalin-fixed and paraffin-embedded tissue sections were isolated and extracted following the TIANamp Genomic DNA kit (QIAGEN, Hilden, Germany) instructions. DNA concentration was quantified by measuring absorbance at 260 nm using a Nanodrop spectrophotometer (ND2000; Thermo Scientific, Waltham, MA, USA). Genomic DNA was divided into 2 identical sections: section A for HpaII treatment and section $\mathrm{B}$ for non-HpaII treatment. Both reaction systems were incubated at $37^{\circ} \mathrm{C}$ for $12 \mathrm{~h}$, followed by heat inactivation of HpaII at $85^{\circ} \mathrm{C}$ for $15 \mathrm{~min}$. The digested DNA was used for polymerase chain reaction (PCR) amplification or stored at $-80^{\circ} \mathrm{C}$.

\section{Detection of methylation level}

PCR primers were designed using DNAMAN (Lynnon Biosoft, San Ramon, CA, USA). The digested DNA was amplified using 2-round PCR. The methylation levels of selected genes were detected using the CCP-based FRET. Ninety microliters CCP working solution and $10 \mu \mathrm{L}$ amplified sample were mixed on a 96-well plate. Absorbance on the 96well plate was then read using the Multi-Mode Microplate Reader (BioTek, Winooski, VT, USA) at an excitation wavelength of $380 \mathrm{~nm}$ and emission wavelengths of 424 and $530 \mathrm{~nm}$. Emission filter $1(440 / 30 \mathrm{~nm})$ was used to detect emission at $424 \mathrm{~nm}$, and emission filter 2 $(528 / 20 \mathrm{~nm})$ was used to detect emission at $530 \mathrm{~nm}$. We calculated FRET ratios $\left(\mathrm{I}_{530 \mathrm{~nm}} / \mathrm{I}_{424 \mathrm{~nm}}\right)$ to determine the methylation level "E" as shown:

$$
\mathrm{E}=\frac{\text { FRET ratio }_{\text {HpaII }}-\text { FRET ratio }}{\text { blank }}
$$


The methylation level was clustered into 3 sections, and each section was assigned a value of $(-),(+)$, or $(2+)$. Value $(-)$ represented a low methylation level below 0.3 , value $(+)$ represented a moderate methylation level from $0.3-0.7$, and value $(2+)$ represented a high methylation level above 0.7 . For each sample, the 3 values were summed.

\section{Statistical analysis}

The SPSS version 19.0 software was used for statistical analysis (SPSS, Inc., Chicago, IL, USA). The association between the methylation level, CA125, and clinical and pathological characteristics was evaluated using a chi-square test. The level of statistical significance was set at $\mathrm{P}<0.05$.

\section{RESULTS}

Based on published literature, 3 tumor suppressor genes, RASSF1A, OPCML, and HOXA9, were selected. Promoter methylation of these genes was observed in high frequency, was stable in all histological types of EOC, and played an important role in ovarian carcinogenesis (Table 1).

Table 1. Genes selected for methylation analysis in EOC by CCP-based FRET technique.

\begin{tabular}{llccll}
\hline Gene name & Chromosome location & Methylation in EOC & Histological type & Assay & Reference \\
\hline RASSF1A & 3 p21.3 & $49 \%(23 / 47)$ & S, M, C, E & MSP & (Wu et al., 2007) \\
& & $58 \%(50 / 86)$ & S, M, C, E & MSP & (Bhagat et al., 2012) \\
OPCML & 7 p15-p14 & $78.4 \%(80 / 102)$ & S, M, C, E & Quantitative MSP & (Zhou et al., 2014) \\
& & $83.3 \%(20 / 24)$ & S, M, C, E & Nested MSP & (Zhou et al., 2011) \\
HOXA9 & $11 \mathrm{q} 25$ & $51 \%(26 / 51)$ & S, M, C, E & MSP & (Wu et al., 2007) \\
& & $95 \%(75 / 79)$ & S* & Headloop MSP & (Montavon et al., 2012) \\
\hline
\end{tabular}

$\mathrm{S}=$ serous carcinoma $\mathrm{M}=$ mucinous carcinoma $\mathrm{C}=$ clear cell carcinoma $\mathrm{E}=$ endometrioid carcinoma; $\mathrm{MSP}=$ methylation-specific PCR. *We only detected high-grade serous ovarian cancer.

CCP-based FRET was used to determine the methylation patterns in DNA isolated from 35 EOC patients with complete clinical pathological information and 11 normal samples. Normal samples showed no methylation in RASSF1A and HOXA9 and 2 moderate methylations in $O P C M L$. Thus, the sum of the 2 normal sample was $(+)$. EOC samples showed increased methylated loci compared to normal samples, with 17 tumor samples showing methylation in RASSF1A, 27 in OPCML, and 27 in HOXA9. There were 32 [91.4\%, sum $\geq(+)]$ tumor samples showing methylation of at least 1 gene. Compared to the sum of the normal samples, the individual gene threshold was set as $(+)[\geq(+)]$, while the sum threshold of EOC samples was set as $(2+)$ [sum $\geq(2+)]$. Consequently, the sensitivity and specificity of the protocol for diagnosing EOC was $85.7 \%$ (30/35) and 100\% (11/11), respectively. CA125 levels in 11 patients corresponding to 11 normal ovarian tissues were all less than $200 \mathrm{U} / \mathrm{mL}$, and those in 25 of $35 \mathrm{EOC}$ patients were more than $200 \mathrm{U} / \mathrm{mL}$, including $2 \mathrm{EOC}$ patients with a sum of $(+)$ and 1 EOC patient with a sum of (-) (Figure 1).

Across all genes analyzed, all groups showed methylation of the 3 genes. Hypermethylation of the $O P C M L$ gene was significantly associated with stage, histological type, grade, and ascites, while that of the RASSF1A and HOXA9 genes showed no correlation with clinico- 
pathological features. The sum was significantly associated with stage and ascites. The same trend was observed for grade. No association was observed between methylation frequency and CA125 level (Table 2).

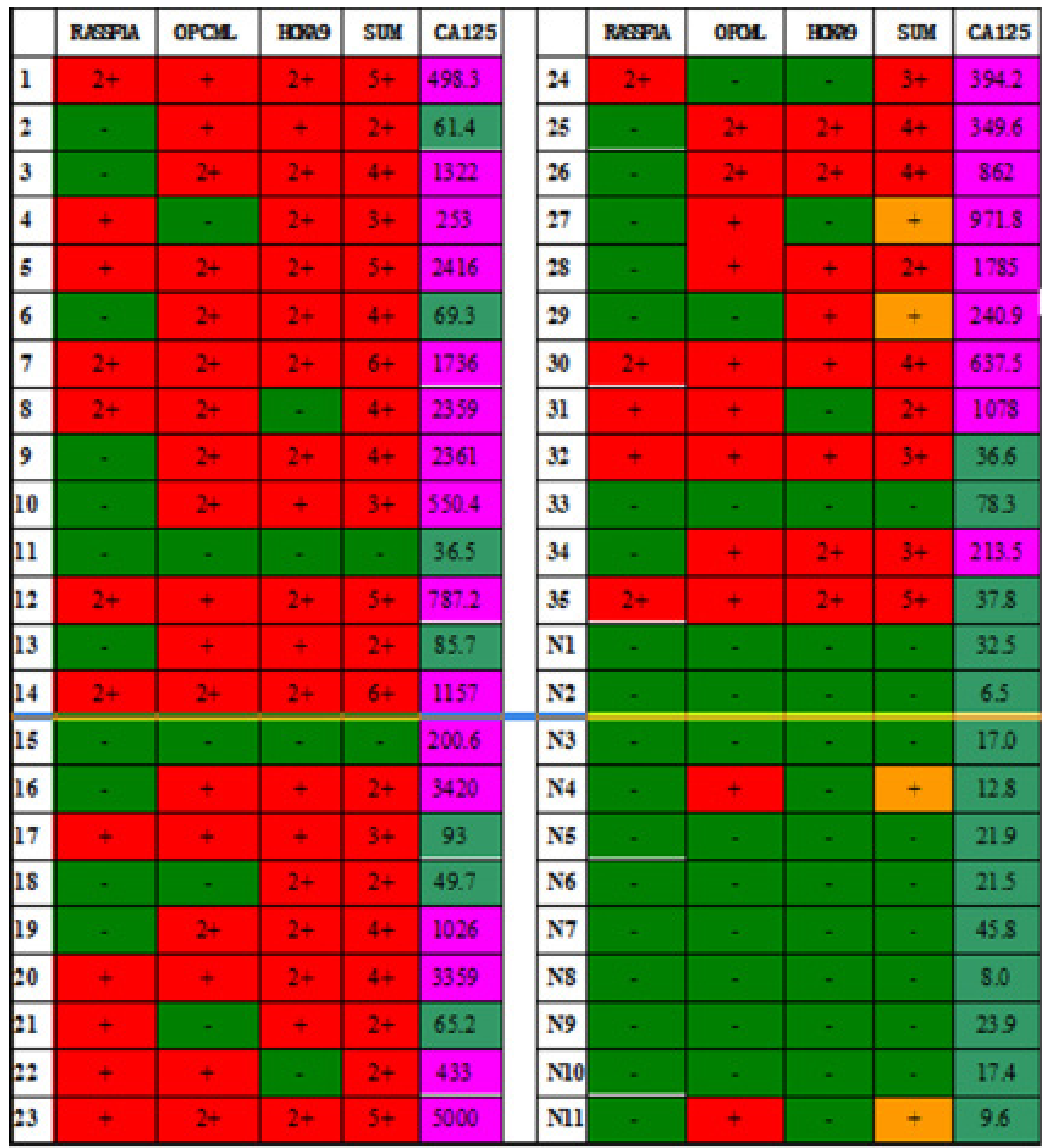

Figure 1. Values of RASSF1A, OPCML, and HOXA9 genes, the sums of 3 genes, and levels of CA125 (U/mL). Thirty-five cancer samples are labeled from 1-35, and 11 normal samples are labeled from N1-N11.

The sensitivity and specificity of the sum for diagnosing EOC were higher than the values for the individual genes. CA125 showed a sensitivity of $71.4 \%$ and a specificity of $100 \%$. However, combining CA125 levels with methylation status for the sum increased the sensitivity to $94.3 \%$ (Table 3 ). 


\begin{tabular}{|c|c|c|c|c|c|}
\hline & RASSFIA & OPCML & HOXA9 & Sum & CA125 > 200 \\
\hline \multicolumn{6}{|l|}{ Age group } \\
\hline$<60$ years & $13 / 28(46.4 \%)$ & $23 / 28(82.1 \%)$ & $21 / 28(75.0 \%)$ & $24 / 28(85.7 \%)$ & $19 / 28(67.9 \%)$ \\
\hline$\geq 60$ years & 4/7 (57.1\%) & $4 / 7(57.1 \%)$ & $6 / 7(85.7 \%)$ & 6/7 (85.7\%) & $6 / 7(85.7 \%)$ \\
\hline$P$ value & 0.612 & 0.165 & 0.552 & 1.000 & 0.357 \\
\hline \multicolumn{6}{|l|}{ FIGO stage } \\
\hline $\mathrm{I} / \mathrm{II}$ & $5 / 11(45.5 \%)$ & $5 / 11(45.5 \%)$ & $7 / 11(63.6 \%)$ & $7 / 11(63.6 \%)$ & $4 / 11(36.4 \%)$ \\
\hline III/IV & $12 / 24(50.0 \%)$ & $22 / 24(91.7 \%)$ & $20 / 24(83.3 \%)$ & $23 / 24(95.8 \%)$ & $21 / 24(87.5 \%)$ \\
\hline$P$ value & 0.806 & 0.010 & 0.204 & 0.045 & 0.002 \\
\hline \multicolumn{6}{|l|}{ Histological type } \\
\hline Serous & $12 / 26(46.2 \%)$ & $22 / 26(84.6 \%)$ & $20 / 26(76.9 \%)$ & $23 / 26(88.5 \%)$ & $22 / 26(84.6 \%)$ \\
\hline Mucous & $2 / 3(66.7 \%)$ & $3 / 3(100 \%)$ & $3 / 3(100 \%)$ & $3 / 3(100 \%)$ & $3 / 3(100 \%)$ \\
\hline Clear cell & $2 / 2(100 \%)$ & $1 / 2(50.0 \%)$ & $2 / 2(100 \%)$ & $2 / 2(100 \%)$ & $0 / 2(0 \%)$ \\
\hline Endometrioid & $1 / 4(25.0 \%)$ & $1 / 4(25.0 \%)$ & $2 / 4(50.0 \%)$ & $2 / 4(50.0 \%)$ & $0 / 4(0 \%)$ \\
\hline$P$ value & 0.241 & 0.030 & 0.262 & 0.206 & 0.00 \\
\hline \multicolumn{6}{|l|}{ Grade of differentiation } \\
\hline High-grade serous cancers & $10 / 21(47.6 \%)$ & $19 / 21(90.5 \%)$ & $17 / 21(81.0 \%)$ & $20 / 21(95.2 \%)$ & $19 / 21(90.5 \%)$ \\
\hline Others & $7 / 14(50.0 \%)$ & $8 / 14(57.1 \%)$ & $10 / 14(71.4 \%)$ & $10 / 14(71.4 \%)$ & $7 / 14(50.0 \%)$ \\
\hline$P$ value & 0.892 & 0.023 & 0.517 & 0.052 & 0.008 \\
\hline \multicolumn{6}{|l|}{ Ascites } \\
\hline Positive & $13 / 24(54.2 \%)$ & $21 / 24(87.5 \%)$ & $20 / 24(83.3 \%)$ & $23 / 24(95.8 \%)$ & $20 / 24(83.3 \%)$ \\
\hline Negative & $4 / 11(36.4 \%)$ & $6 / 11(54.5 \%)$ & $7 / 11(63.6 \%)$ & $7 / 11(63.6 \%)$ & $5 / 11(45.5 \%)$ \\
\hline$P$ value & 0.335 & 0.034 & 0.204 & 0.045 & 0.023 \\
\hline \multicolumn{6}{|l|}{ CA125 } \\
\hline$>200 \mathrm{U} / \mathrm{mL}$ & $13 / 25(52.0 \%)$ & $21 / 25(84.0 \%)$ & $19 / 25(76.0 \%)$ & $22 / 25(88.0 \%)$ & \\
\hline$<200 \mathrm{U} / \mathrm{mL}$ & $4 / 10(40.0 \%)$ & $6 / 10(60.0 \%)$ & $8 / 10(80.0 \%)$ & $8 / 10(80.0 \%)$ & \\
\hline$P$ value & 0.527 & 0.279 & 0.802 & 0.547 & \\
\hline
\end{tabular}

Table 3. Discrimination between EOC and normal ovarian tissues by different models.

\begin{tabular}{lcccr}
\hline & EOC $(\mathrm{N}=35)$ & Normal $(\mathrm{N}=11)$ & Sensitivity & Specificity \\
\hline RASSF1A & 17 & 0 & $48.6 \%$ & $100.0 \%$ \\
OPCML & 27 & 2 & $77.1 \%$ & $81.8 \%$ \\
HOXA9 & 27 & 0 & $77.1 \%$ & $100.0 \%$ \\
Sum & 30 & 0 & $85.7 \%$ & $100.0 \%$ \\
CA125 & 25 & 0 & $71.4 \%$ & $100.0 \%$ \\
Sum + CA125 & 33 & 0 & $94.3 \%$ & $100.0 \%$ \\
\hline
\end{tabular}

\section{DISCUSSION}

In this study, we analyzed the correlation between the methylation status of candidate genes and clinicopathological features in order to confirm the feasibility of CCP-based FRET. Based on published studies, the 3 genes RASSF1A, OPCML, and HOXA9 were selected because they show a high frequency of methylation in EOC and no methylation in normal tissues (Rathi et al., 2002; Teodoridis et al., 2005; Wu et al., 2007; Liggett et al., 2011). In EOC, inactivation of the RASSF1A, OPCML, and HOXA9 gene promoters was reported to be related to carcinogenesis or early cancer (Sellar et al., 2003; Montavon et al., 2012; Bhagat et al., 2012; Zhang et al., 2013).

OPCML is an opioid binding protein cell adhesion molecule. Over-expression of OPCML decreases the proliferation of EOC cells in vitro and tumor growth in vivo while increasing cellular aggregation (Sellar et al., 2003). Zhou et al. (2011) found that the methylation frequency of the $O P C M L$ gene in ovarian cancer samples differed in different assays, with values of $58.3 \%(14 / 24)$ by methylation-specific PCR and $83.3 \%(20 / 24)$ by nested 
methylation-specific PCR, respectively, and no methylation was observed in normal ovarian tissues using either method. In our study, the percentages of promoter hypermethylation of OPCML were $77.1 \%$ in EOC and $18.2 \%$ in normal ovarian tissues. This indicates that our more sensitive method improved the methylation frequency compared with the methods used in other studies (Teodoridis et al., 2005; Chen et al., 2007). Another study found that $O P C M L$ promoter methylation was significantly associated with an older patient age, advanced stage of ovarian cancer, and poor overall survival of ovarian cancer patients (Zhou et al., 2014). Similarly, we found that the methylation of $O P C M L$ was significantly associated with advanced stage, high-grade, serous cancer and mucous cancer, and positive ascites. Loss of OPCML simultaneously reduces intercellular adhesion and accelerates cell growth, not only promoting the early steps of ovarian carcinogenesis but also causing cancer development.

RASSF $1 A$ acts as a tumor suppressor gene in the RAS pathway and can regulate proliferation, induce apoptosis, and bind to and stabilize microtubules (Liu et al., 2005). Previous studies showed that the frequency of promoter hypermethylation of RASSF1A in ovarian carcinomas was 40-58\% (Yoon et al., 2001; Bhagat et al., 2012). In our study, epithelial ovarian cancers exhibited RASSF1A gene promoter methylation frequencies of $48.6 \%$ (17/35). Ibanez de Caceres et al. (2004) reported a significant correlation between methylation status, tumor grade, CA125 levels, and stage in ovarian cancer. However, we found that the methylation status of RASSF1A was not related to age, grade, stage, histological type, ascites, and CA125, which was similar to the results of Bhagat et al. (2012). Homeodomain-containing genes encode transcriptional factors that function during embryonic development to control patterning, differentiation, and proliferation (Gorski and Walsh, 2003). A study by Montavon et al. (2012) found a 95\% frequency of methylation of HOXA9 in high-grade serous ovary cancer, as HOXA9 regulates serous differentiation of the Müllerian ducts to Fallopian tubes (Du and Taylor, 2004; Chen et al., 2005) and HOXA9 methylation in ovarian cancer may reflect a loss of transcriptional plasticity during disease development and a shift towards epithelial cell dedifferentiation or high-grade classification (Coolon et al., 2010). Our study used CCP-based FRET to detect the 4 types of EOC. Methylation frequency of the $H O X 9$ gene was $77.14 \%$, which was higher than the value of $51 \%(26 / 51)$ by methylation-specific PCR found in a previous report (Wu et al., 2007). There was no significant association between gene methylation and the high grade of EOC, but methylation frequency in the high-grade serous ovarian cancers $(81 \%)$ was higher than that of other EOCs $(71 \%)$. The small number of samples in our study may account for this trend. No normal ovarian samples were included in the methylation of RASSF $1 A$ and HOXA9.

In our study, the technique for detecting methylation was more sensitive and the methylation status of 3 genes agreed with previously reported values, although different techniques such as methylation-specific PCR were used in these studies. The thresholds, $(+)$ for individual genes and (2+) for the sum, were reasonable. Our protocol is advantageous because it takes the degree and contribution of promoter methylation in different candidate gene into account. The methylation of $O P C M L$ was significantly associated with stage, histology type, grade and ascites, while RASSF1A and HOXA9 were not. The sum could be used for comprehensive analysis, exhibiting a significant association with stage, ascites, and grade, but no relevance with histological type. The sum also balanced the different effects of methylation of individual gene in specificity and improved the sensitivity and specificity of the method.

The sum of all normal samples was less than $(+)$, and 30 of 35 cancer samples exceeded $(+)$. Thus, a sum of $\geq(2+)$ was set as a threshold. If the sum of a sample exceeded $(+)$, 
cancer diagnosis was confirmed. The sensitivity of the protocol was calculated to be $85.7 \%$ (30/35), with a specificity of $100 \%(11 / 11)$. However, 4 samples showed a sum of (+), with 2 samples in the cancer group and 2 in the normal group. The sum of $(+)$ was borderline, making it difficult to determine whether the disease was cancerous or benign. To assess whether gene methylation could be used to improve existing molecular biomarkers, we investigated the discrimination properties of pre-operative serum CA125 levels in our study. CA125 level was not related to the sum of methylation level. Combining CA125 with the methylation level sum revealed 2 EOC cases whose sums were (+) and 1 EOC case whose sum was (-). The sensitivity increased to $94.3 \%$ (33/35), while specificity remained at $100 \%$.

Recent studies have shown that aberrant DNA methylation, which typically occurs before patients develop clinical manifestations and radiographic evidence, can be detected in cellfree serum (Vlassov et al., 2010; Liggett et al., 2011; Zhang et al., 2013). The amount of available cell-free serum DNA is often very low. The CCP-based FRET technique is sensitive, thus small DNA samples obtained from serum, plasma, or stool can be examined. This method can also detect low methylation levels of the gene promoter, meeting the requirement for early cancer diagnosis. In the future, we will continue to examine this protocol for detecting early EOC using cell-free serum DNA and investigate the potential application in monitoring EOC treatment.

\section{Conflicts of interest}

The authors declare no conflict of interest.

\section{ACKNOWLEDGMENTS}

The authors express their sincere gratitude to Prof. Wang and Dr. Zhang of the Chinese Academy of Sciences, Beijing, China, for help with CCP-based FRET. Research supported by the Science and Technology Development Foundation of Nanjing Medical University (\#2012NJMU094).

\section{REFERENCES}

Bast RC Jr, Badgwell D, Lu Z, Marquez R, et al. (2005). New tumor markers: CA125 and beyond. Int. J. Gynecol. Cancer 15 (Suppl 3): 274-281.

Bhagat R, Chadaga S, Premalata CS, Ramesh G, et al. (2012). Aberrant promoter methylation of the RASSF1A and APC genes in epithelial ovarian carcinoma development. Cell. Oncol. 35: 473-479.

Chen H, Ye F, Zhang J, Lu W, et al. (2007). Loss of OPCML expression and the correlation with CpG island methylation and LOH in ovarian serous carcinoma. Eur. J. Gynaecol. Oncol. 28: 464-467.

Cheng W, Liu J, Yoshida H, Rosen D, et al. (2005). Lineage infidelity of epithelial ovarian cancers is controlled by HOX genes that specify regional identity in the reproductive tract. Nat. Med. 11: 531-537.

Coolen MW, Stirzaker C, Song JZ, Statham AL, et al. (2010). Consolidation of the cancer genome into domains of repressive chromatin by long-range epigenetic silencing (LRES) reduces transcriptional plasticity. Nat. Cell Biol. 12: $235-246$.

Cul'bová M, Lasabová Z, Stanclová A, Tilandyová P, et al. (2011). Methylation of selected tumor-supressor genes in benign and malignant ovarian tumors. Ceska Gynekol. 76: 274-279.

Du H and Taylor HS (2004). Molecular regulation of Mullerian development by Hox genes. Ann. NY Acad. Sci. 1034: $152-165$

Egger G, Liang G, Aparicio A and Jones PA (2004). Epigenetics in human disease and prospects for epigenetic therapy. Nature 429: 457-463.

Gorski DH and Walsh K (2003). Control of vascular cell differentiation by homeobox transcription factors. Trends 
Cardiovasc. Med. 13: 213-220.

Ho CM, Huang CJ, Huang CY, Wu YY, et al. (2012). Promoter methylation status of HIN-1 associated with outcomes of ovarian clear cell adenocarcinoma. Mol. Cancer 11: 53.

Ibanez de Caceres I, Battagli C, Esteller M, Herman JG, et al. (2004). Tumor cell-specific BRCA1 and RASSF1A hypermethylation in serum, plasma, and peritoneal fluid from ovarian cancer patients. Cancer Res. 64: 6476-6481.

Issa JP (2007). DNA methylation as a therapeutic target in cancer. Clin. Cancer Res. 13: 1634-1637.

Jacobs IJ and Menon U (2004). Progress and challenges in screening for early detection of ovarian cancer. Mol. Cell Proteomics 3: 355-366.

Jemal A, Siegel R, Xu J and Ward E (2010). Cancer statistics. 2010. CA Cancer J. Clin. 60: 277-300.

Kurman RJ (2013). Origin and molecular pathogenesis of ovarian high-grade serous carcinoma. Ann. Oncol. 24 (Suppl 10): $\mathrm{x} 16-\mathrm{x} 2$.

Landen Jr CN, Birrer MJ and Sood AK (2008). Early events in the pathogenesis of epithelial ovarian cancer. J. Clin. Oncol. 26: 995-1005.

Liggett TE, Melnikov A, Yi Q, Replogle C, et al. (2011). Distinctive DNA methylation patterns of cell-free plasma DNA in women with malignant ovarian tumors. Gynecol. Oncol. 120: 113-120.

Liu L, Broaddus RR, Yao JC, Xie S, et al. (2005). Epigenetic alterations in neuroendocrine tumors: methylation of RASassociation domain family 1, isoform A and p16 genes are associated with metastasis. Mod. Pathol. 18: 1632-1640.

Menon U and Jacobs IJ (2000). Recent developments in ovarian cancer screening. Curr. Opin. Obstet. Gynecol. 12: 39-42.

Montavon C, Gloss BS, Warton K, Barton CA, et al. (2012). Prognostic and diagnostic significance of DNA methylation patterns in high grade serous ovarian cancer. Gynecol. Oncol. 124: 582-588.

Munkarah A, Chatterjee M and Tainsky MA (2007). Update on ovarian cancer screening. Curr. Opin. Obstet. Gynecol. 19: 22-26.

Rathi A, Virmani AK, Schorge JO, Elias KJ, et al. (2002). Methylation profiles of sporadic ovarian tumors and nonmalignant ovaries from high-risk women. Clin. Cancer Res. 8: 3324-3331.

Sellar GC, Watt KP, Rabiasz GJ, Stronach EA, et al. (2003). OPCML at 11q25 is epigenetically inactivated and has tumorsuppressor function in epithelial ovarian cancer. Nat. Genet. 34: 337-343.

Teodoridis JM, Hall J, Marsh S, Kannall HD, et al. (2005). CpG island methylation of DNA damage response genes in advanced ovarian cancer. Cancer Res. 65: 8961-8967.

Vlassov VV, Laktionov PP and Rykova EY (2010). Circulating nucleic acids as a potential source for cancer biomarkers. Curr. Mol. Med. 10: 142-165.

Widschwendter M, Apostolidou S, Jones AA, Fourkala EO, et al. (2009). HOXA methylation in normal endometrium from premenopausal women is associated with the presence of ovarian cancer: a proof of principle study. Int. $J$. Cancer 125: 2214-2218.

Wu Q, Lothe RA, Ahlquist T, Silins I, et al. (2007). DNA methylation profiling of ovarian carcinomas and their in vitro models identifies HOXA9, HOXB5, SCGB3A1, and CRABP1 as novel targets. Mol. Cancer 6: 45.

Yoon JH, Dammann R and Pfeifer GP (2001). Hypermethylation of the CpG island of the RASSF1A gene in ovarian and renal cell carcinomas. Int. J. Cancer 94: 212-217.

Zhang J, Xing B, Song J, Zhang F, et al. (2014). Associated analysis of DNA methylation for cancer detection using CCPbased FRET technique. Anal. Chem. 86: 346-350.

Zhang Q, Hu G, Yang Q, Dong R, et al. (2013). A multiplex methylation-specific PCR assay for the detection of earlystage ovarian cancer using cell-free serum DNA. Gynecol. Oncol. 130: 132-139.

Zhou F, Cao X, Liu M, Wang Y, et al. (2011). A study of the methylation status of opioid binding protein/cell adhesion molecule-like gene in ovarian cancer using nested methylation-specific polymerase chain reaction detection. Clin. Lab. 57: 421-424.

Zhou F, Tao G, Chen X, Xie W, et al. (2014). Methylation of OPCML promoter in ovarian cancer tissues predicts poor patient survival. Clin. Chem. Lab. Med. 52: 735-742.

Zhu J and Yao X (2007). Use of DNA methylation for cancer detection and molecular classification. J. Biochem. Mol. Biol. 40: 135-141. 